\title{
Deutsch-jüdische Autobiographie
}

Markus Malo

\section{Zur Theorie und Gattungsgeschichte autobiographischer Texte}

Die Autobiographie als ursprünglich den rhetorischen Zweckformen zugeordnete Gattung ist erst im 18. Jahrhundert in den Rang einer literarischen Gattung aufgestiegen. Sie hat sich in der kurzen Zeit ihrer Existenz häufig gewandelt und wurde von der ihre Entwicklung begleitenden Literaturwissenschaft deshalb unterschiedlich gedeutet. Dies erschwert eine über das Minimale hinausgehende Definition; die verschiedenen Definitionsversuche beschränken sich daher auf das Kriterium der wie auch immer literarisch überformten - Wirklichkeitsaussage als Wesenskern der Autobiographie, durch den diese sich vom Roman, an dessen formaler Entwicklung sie sich orientiert, unterscheidet. Dagegen sollen hier die historische Entwicklung und Erforschung der Gattung im deutschsprachigen Raum skizziert werden - als Folie für die Darstellung des ,Sonderwegs‘ der deutschsprachigen jüdischen Autobiographie. Ausführliche Überblicksdarstellungen über Geschichte und Theorie der Autobiographie gibt es u.a. von Michaela Holdenried (2000) und Martina WagnerEgelhaaf (2000).

Für die frühe ,Geistesgeschichte' und die Geschichtswissenschaft stellt die Autobiographie vor allem eine Wirklichkeitsaussage dar, aus der sich unmittelbare Aufschlüsse über das Leben und die geistige Entwicklung, aber auch die äußeren Lebensumstände des autobiographischen Ichs gewinnen lassen. Diese Annahme ist vor allem von der idealistischen Subjektphilosophie und ihrer Annahme einer personalen Identität geprägt, die spätestens zu Beginn des 20. Jahrhunderts philosophisch in Frage gestellt wurde. Obwohl die Geisteswissenschaften bis heute die Autobiographie als vor allem sozialgeschichtlich relevante Quelle schätzen und nutzen, hat die Geschichtswissenschaft schon zu Beginn des 20. Jahrhunderts den Quellenwert der Autobiographie kritisch eingeschätzt (vgl. Glagau 1903).

Die zunehmende Skepsis der Geschichtswissenschaft liegt in einer Veränderung der autobiographischen Gattung im späten 18. Jahrhundert begründet, die sich tatsächlich von einer rhetorischen Zweckform, mit der das eigene Leben zunächst religiös, später dann zunehmend weltlich (vgl. Niggl 1997 und Lehmann 1988) legitimiert wird und den Nachgeborenen Kunde davon gegeben werden sollte, hin zu einer immer stärker ,literarisierten', am Bildungsroman der Zeit orientierten Form entwickelt, die Elemente des fiktionalen Erzählens übernommen und gattungspoetologische und ästhetische Überlegungen zur Grundlage des autobiographischen Schreibens erhoben hat (vgl. Müller 1976, Lehmann 1988). Zugleich sind nachprüfbare Fakten beim autobiographischen Schreiben in den Hintergrund gerückt, weil vermehrt Bildungsprozesse und Seelenzustände ins Blickfeld der Verfasser geraten sind. 
Damit ist die Autobiographie zum Gegenstand der Literaturwissenschaft geworden, die sich seit der Mitte des vergangenen Jahrhunderts sowohl neuen Methoden der Forschung als auch neuen Gegenständen zugewendet hat. Für die Autobiographie bedeutet das, dass sie jetzt weniger unter, inhaltlichen' Aspekten und hinsichtlich ihres Quellenwerts untersucht wird, sondern dass ihre Form in geographischen und historischen Zusammenhängen diachron und synchron vermessen und von anderen Formen von Selbstzeugnissen - zu nennen sind hier etwa Brief, Memoiren und Tagebuch - sowie von den fiktionalen Formen abgegrenzt wird (vgl. Niggl 1998).

Diese Definitionsversuche waren jedoch nicht von dauerhaftem Erfolg, weil sich die traditionellen Identitätsannahmen unter den Bedingungen der Moderne als illusionär erwiesen hatten und die Konstruktion des autobiographischen Ichs - die bislang als eine Konstante autobiographischer Selbstvergewisserung galt - zunehmend selbst als Fiktion entlarvt wurde. Zuerst hat dies wohl Paul de Man 1979 in seinem Aufsatz Autobiographie als Maskenspiel unternommen, der die Autobiographie wesentlich als „Verstehensfigur“ und nicht als durch bestimmte formale Elemente konstituierte literarische Gattung definierte. Damit wurde auch die Trennung von autobiographischem und fiktionalem Schreiben in der Autobiographieforschung unhaltbar. Dies bedeutete eine Ausweitung des zu untersuchenden Textkorpus ins nahezu Unermessliche, weil nun auch Identitätskonstruktionen und Erinnerungsmechanismen in bislang als fiktional aus dem autobiographischen Kanon ausgeschlossenen Werken untersucht werden konnten (Holdenried 1991, Sill 1991, Günter 1996). Die jüngere Forschung löst sich deshalb vom AutobiographieBegriff, weil sie das autobiographische Schreiben weniger als eine literarische Gattung mit klar umrissenen Konturen, sondern vielmehr als ein Rezeptionsphänomen betrachtet, das sich am ehesten unter dem mit weniger begriffsgeschichtlichem Ballast behafteten Begriff der „Autofiktion“ fassen lässt (Gasser 2012).

Dagegen steht der französische Literaturwissenschaftler Philippe Lejeune, der einen produktions- und rezeptionsästhetisch motivierten „autobiographischen Pakt“ zwischen Leser und Verfasser postuliert. Darin stützt er sich auf philosophische und identitätslogische Prämissen und lässt die Formfrage offen. Damit bleibt die Autobiographie eine Form der ,subjektiven Geschichtsschreibung، (Sandberg 2000), die ihre narrative Konstruiertheit nicht verbirgt. Dies hängt mit einem gewandelten Selbstverständnis auch der Geschichtswissenschaft zusammen, in der sich die Erkenntnis durchgesetzt hat, dass auch sie letztlich narrativ verfährt und vergangene Wirklichkeit höchstens ausschnittweise, interessengeleitet und mittelbar darzustellen vermag und sich hierzu Verfahren bedient, die auch in den fiktionalen Textgattungen verwendet werden (White 1986). In der neueren Literaturwissenschaft hat umgekehrt der ,New Historicism“ auf die „Geschichtlichkeit der Texte und der Textualität der Geschichte“ (Montrose 1995, 67) hingewiesen und damit eine unhintergehbare Reziprozität von Text und Wirklichkeit postuliert. 
Um diesen definitorischen Dilemmata zu entgehen, sollen im Folgenden, basierend auf Lejeunes These des autobiographischen Pakts zwischen Autor und Leser, wesentliche Motive der deutschsprachigen jüdischen Autobiographie sowie exemplarische Texte vorgestellt werden, um zum einen wesentliche Konstituenten der Gattung herauszuarbeiten, zum anderen aber ihre Geschichtlichkeit und Veränderlichkeit über die Zeitläufte hinweg zu demonstrieren.

\section{Die deutschsprachige jüdische Autobiographie}

Die Geschichte des Judentums in Deutschland seit der Aufklärung bis weit ins 20. Jahrhundert hinein ist eine solche der Akkulturation und Assimilation von jüdischer Seite bzw. ihrer Zurückweisung durch die Mehrheitsgesellschaft. Dies zeigt sich auch beim Blick auf die deutschsprachige jüdische Autobiographie, die in der literarischen Gestaltung entweder mit der Autobiographie der christlichen Mehrheitsgesellschaft konform geht oder dieser sogar neue Impulse gibt (Günter 1996). Die spezifische Differenz ist also nicht in der literarischen Form, sondern im kulturellen Hintergrund und den verhandelten Themen zu suchen. Ziel einer Darstellung der deutsch-jüdischen Autobiographie ist also mit Andreas Kilcher „,zu fragen, mit welchen argumentativen Strategien in den verschiedenen historischen Diskursen [...] der irreduzibel vieldeutige interkulturelle Raum der deutsch-jüdischen Literatur konstruiert und interpretiert wird“ (Kilcher 1999, 511).

Da die Autobiographie eben nicht nur eine literarische Gattung mit dem Ziel der Publikation, sondern darüber hinaus eine rhetorische, häufig nur für den ,Hausgebrauch' abgefasste Gattung mit starkem Wirklichkeitsbezug und legitimatorischem Charakter ist, sei an dieser Stelle zumindest darauf hingewiesen, dass Autobiographien von Juden aus Deutschland in nahezu allen Sprachen der Welt und in allen Kulturkreisen geschrieben worden sind. Dies liegt in den verschiedenen Emigrationswellen aus Deutschland begründet, die im 19. Jahrhundert vor allem ökonomische Gründe hatten. Mit der Verfolgung und Vertreibung der europäischen Juden durch den Nationalsozialismus hat sich die ursprünglich deutschsprachige jüdische Autobiographie mit ihren kulturellen deutschen und jüdischen Hintergründen weltweit verbreitet. Des Weiteren hat der vermutlich größte Teil der existierenden Autobiographien nie das Licht der Öffentlichkeit erblickt und liegt, sofern zwischenzeitlich keine Digitalisierung oder auszugsweise Veröffentlichung der Inhalte wie beim Bestand des Leo-Baeck-Institute stattgefunden hat (vgl. Monika Richarz 1976-1982 und die digitale Präsentation unter digibaeck), noch immer weitgehend unbeachtet in öffentlichen (z.B. die Sammlung der Houghton Library in Harvard) oder privaten Archiven.

Jenseits der Rekonstruktion der Diskurse ,deutsch-jüdischer‘ Interkulturalität, die Andreas Kilcher als konstitutiv für den Begriff der deutsch-jüdischen Literatur und somit auch der deutsch-jüdischen Autobiographie ansieht (Kilcher 1999, 511), 
hat Christoph Miething versucht, den ,Wesenskern“ der jüdischen Autobiographie als spezifische Differenz zur christlich motivierten Autobiographik zu benennen. Steht in der christlichen Autobiographie der Gegensatz von Außenwelt und Innenwelt und die Einmaligkeit des Individuums in Auseinandersetzung mit den Umgebungsvariablen Geschichte und Gesellschaft im Zentrum, ist in der jüdischen Autobiographie „eine andere Konstruktion von Individualität erkenn[bar]“ (Miething 2003, 52). Hier ist die Einbettung des Individuums in die traditionellen und historischen Zusammenhänge der kollektiven jüdischen Identität von primärem Interesse, während in der christlichen Autobiographie das Heraustreten des Einzelnen aus den überindividuellen Zusammenhängen im Fokus steht, wie die programmatischen Worte Goethes in der Einleitung zu Dichtung und Wahrheit belegen: „Denn dieses scheint die Hauptaufgabe der Biographie zu sein, den Menschen in seinen Zeitverhältnissen darzustellen und zu zeigen, inwiefern ihm das Ganze widerstrebt, inwiefern es ihn begünstigt, wie er sich eine Welt- und Menschensicht daraus gebildet und wie er sie, wenn er Künstler, Dichter, Schriftsteller ist, wiederum nach außen abgespiegelt" (Goethe 1985, 11).

Die deutschsprachige jüdische Autobiographie ist wesentlich bestimmt von Diskursen über die kollektive jüdische Identität, die als komplexes Gemisch von Eigen- und Fremdwahrnehmung entstanden ist. Sie ist gekennzeichnet durch das allmähliche Heraustreten des Individuums aus dieser kollektiven Identität. In ihr spiegelt sich das ambivalente Verhältnis ihrer Autorinnen und Autoren zur Kultur der christlichen deutsch(sprachig)en Mehrheitsgesellschaft und der durch die Mehrheitsgesellschaft vorgenommenen Fremdzuschreibungen einer jüdischen Identität, gegen die eine individuelle Identität erst errungen und behauptet werden muss. Die aus diesem Phänomen resultierende Erfahrung von Alterität und der produktive Umgang damit sind konstitutiv für die deutsch-jüdische Autobiographie. Dass umgekehrt die deutschsprachige jüdische Autobiographie vor allem durch die Darstellung des Umgangs mit antijüdischen und antisemitischen Stereotypen und Vorurteilen charakterisiert werden kann, ist natürlich ebenso richtig.

Auffällig oft sehen sich die Verfasser solcher Lebensbeschreibungen nicht in erster Linie als Individuum, dessen Geschichte sie erzählen, sondern als ,Repräsentanten' einer ihnen von außen auferlegten kollektiven Identität und eines kollektiven Schicksals, über deren Folgen sie ihrem Publikum Zeugnis ablegen wollen (vgl. Yerushalmi 1996). Mit dieser Fremdzuschreibung von Identität geht die Erfahrung der Kontingenz einher, die häufig als lebensbestimmend empfunden wird, weil diese vom Individuum wie von der Gemeinschaft nicht kontrollierbaren Fremdzuschreibungen Folgen für den äußeren und den inneren Lebensweg des Individuums haben.

Urszene der jüdischen Geschichtserinnerung ist der Seder-Abend des PessachFestes, an dem die Erinnerung an die Befreiung aus der ägyptischen Gefangenschaft zelebriert wird. Dieses Fest bekommt in zahlreichen jüdischen Autobiographien, vor allem aber in denjenigen aus der Generation der Holocaust-Überlebenden, eine prominente Bedeutung; gegenwärtige Erfahrungen werden auf diese Urszene jüdi- 
scher Verfolgungs- und Errettungsszenarien projiziert: „Manches davon, was uns Berliner Kindern damals so fern schien, hat uns im Leben eingeholt; Moses Errettung in dem Kästlein aus Rohr, die Knechtschaft in Ägypten und die Plagen [...]“ (Greve 1994, 33).

Das Gebot „Gedenk! Erinnere dich! Thiskor!“ (Sperber 1983, 69) ist daher ein zentrales Movens jüdischer Autobiographik in deutscher Sprache und mahnt nicht nur zur subjektiven Selbstvergewisserung, wie sie in der christlichen Autobiographie so bedeutsam ist, sondern vor allem zur kollektiven Erinnerungsarbeit.

\section{Geschichte der deutschsprachigen jüdischen Autobiographie - eine Skizze}

\section{Tradition und Emanzipation: Die Anfänge der deutsch-jüdischen Autobiographie}

Als erste deutschsprachige jüdische Autobiographie können die Denkwürdigkeiten der jüdischen Kaufmannsfrau Glückel (Glikl) von Hameln (1646-1724) gelten, die ihre Erinnerungen an ihr Leben in zwei verschiedenen Abschnitten ihres Lebens zu Papier brachte. Um 1690 schrieb sie die ersten fünf Bücher nieder, die ihre und ihrer Familie Lebensgeschichte von Glückels Geburt bis zum Tod ihres ersten Mannes, des erfolgreichen Kaufmanns Chajim umfassen. Die beiden letzten Bücher thematisieren Glückels späte Jahre mit ihrem zweiten Mann Cerf Levy, einem reichen jüdischen Bankier in Metz, der nach einem Bankrott starb und seine Witwe in absoluter Mittellosigkeit zurückließ, sodass Glückel bei ihrer Tochter unterkommen musste. Mit ihrer Schilderung des jüdischen Alltagslebens in Hamburg und Metz stellt Glückels Text damit eine nicht hoch genug zu schätzende Quelle für das jüdische Gemeindeleben ihrer Zeit dar. Dabei beschränkt sich Glückel nicht nur auf die äußeren Ereignisse ihres ungewöhnlich langen Lebens, sondern berichtet auch über die politische und soziale Wirklichkeit ihrer Zeit, insoweit sie ihre eigene Lebenswirklichkeit berühren. Abgerundet wird der Text durch moralische Erzählungen und Zitate aus Tora und nachbiblischen jüdischen Schriften, die den Adressaten ihres Textes, ihre Kinder und deren Nachkommen, leiten sollen: „Meine lieben Kinder, ich gehe nicht darauf aus, euch ein Moralbuch zu machen und zu schreiben [...]. Wir haben unsere heilige Thora, damit wir alles daraus ersehen und begreifen können, was uns nützlich ist und was uns vom Diesseits in das Leben des Jenseits bringt“ (Glückel 1910, 11).

Die Autobiographie Glückels zeichnet sich dadurch aus, dass sie, noch vor der jüdischen Emanzipationsbewegung seit dem Ende des 18. Jahrhunderts, an der Schwelle zur Moderne verfasst, das traditionelle jüdische Kaufmannsleben aus der Perspektive einer Frau schildert. Als Leitlinie ihres Lebens sieht sie dabei ihre Gesetzestreue und Schicksalsergebenheit an, deren Nachahmung sie ihren Nachkommen empfiehlt. Damit stellt Glückel ihre Autobiographie in die Tradition des ethi- 
schen Testaments, der tsava ,ah (vgl. Miething, 2003, 53). Der Text ist in Jiddisch abgefasst und mit hebräischen Lettern geschrieben. Seine Intention ist es, Exempel für die eigenen Nachkommen zu sein. Erstmals im Original veröffentlicht wurde er 1896, zwei deutsche Übersetzungen erschienen 1910 und 1913, d.h. der Text hat erst sehr spät eine gewisse Bekanntheit und Wirkungsmacht entwickelt.

Hat Glückel noch ausschließlich für die eigene Familie geschrieben, wendet sich Salomon Maimons (um 1753-1800) auf deutsch 1792/93 verfasste und von Karl Philipp Moritz herausgegebene Lebensgeschichte an ein anonymes, breites Publikum, um ihm ein Exempel für ein Leben im ,Licht‘ der Aufklärung zu geben, wie der Herausgeber der Neuausgabe betont (Maimon 1995, 8).

Mit diesem Text ist die deutsch-jüdische Autobiographie in der deutschen und europäischen Literaturgeschichte angekommen, weil mit Maimon erstmals ein mit der literarischen Tradition vertrauter, sich mit der christlichen Mehrheitskultur auseinandersetzender jüdischer Autobiograph an die Öffentlichkeit tritt. Maimons Lebensgeschichte orientiert sich an den autobiographischen Texten von Jung-Stilling, Moritz und Rousseau, steht aber auch in der Tradition der Gelehrtenautobiographie, weil vor allem der intellektuelle Werdegang des Protagonisten geschildert wird: „Ich bin aus Polen gebürtig, von der jüdischen Nation, nach meiner Erziehung und meinem Studium zum Rabbiner bestimmt, habe aber in der dicksten Finsternis einiges Licht erblickt. Dies bewog mich, nach Licht und Wahrheit weiter zu forschen und mich aus der Finsternis des Aberglaubens und der Unwissenheit völlig loszumachen; zu diesem Zwecke ging ich [...] nach Berlin, wo ich durch Unterstützung einiger aufgeklärter Männer unserer Nation einige Jahre studierte“ (Maimon 1995, 183).

Maimons Existenz ist von diesem Dualismus - der Ablehnung der rabbinischen Weisheit zugunsten der Vernunftwahrheit der (christlichen) Aufklärung und dem seiner ursprünglichen Inhalte beraubten Habitus des rabbinischen Weisen - geprägt, weswegen es ihm weder gelingt, seinen Platz in der jüdischen Gesellschaft zu behaupten noch in der christlichen Fuß zu fassen. Damit ist inhaltlich ein Grundproblem der Moderne, das Gefühl der Nichtzugehörigkeit durch Entfremdung von den traditionellen, religiös geprägten Lebenszusammenhängen, benannt, das aber erst in der Autobiographie und im Roman des 20. Jahrhunderts seine formale Entsprechung finden sollte.

\section{Enttäuschte Hoffnungen: Das Zeitalter des Liberalismus}

Die zumindest äußerliche Integration in die christliche Mehrheitsgesellschaft sollte erst den auf Maimon folgenden Generationen deutschsprachiger Juden im 19. Jahrhundert gelingen. Ihre Autobiographien unterscheiden sich formal kaum von der nun vorherrschenden Memoirenliteratur, die weniger den intellektuellen Werde- 
gang ihrer Protagonisten als vielmehr die gesellschaftliche Etablierung des ,außengeleiteten Individuums‘ (Neumann 1970) zum Gegenstand der Darstellung macht. Wichtig sind nun nicht mehr Kindheit und Jugend mit ihren Bildungserlebnissen und Erfahrungen, die den ,Lebensweg“ (Vollers-Sauer 1993) des Menschen prägen, sondern berufliche und gesellschaftliche Anerkennung, die das autobiographische Individuum in die Gesellschaft seiner Zeit einordnen und ihm seinen angemessenen ,Rang' zuweisen. Damit wird die Autobiographie zum Verständigungsmittel des Bürgertums über die selbst erbrachten Leistungen. Infolgedessen verliert die Gattung ihren literarischen Charakter wieder, den sie um 1800 gewonnen hat, und wird zum bloßen ,Bericht‘ äußerer Erlebnisse und Ereignisse (vgl. Lehmann 1988).

Als paradigmatisch für eine solche Form der Autobiographie kann die 1854, im Jahre nach seinem Tod veröffentlichte Geschichte meines Dienstlebens des jüdischen Offiziers in preußischen Diensten Meno Burg (1788-1853) gelten, der sich seiner Ausnahmestellung sehr bewusst war. Burg konstruiert seine Lebensgeschichte, die vor allem aus der berichtenden Darstellung der begünstigenden und behindernden Umstände besteht, die ihm die Erreichung seines militärischen ,Ranges‘ ermöglichten, als Exempel: „Sie soll den jüdischen Jüngling ermutigen, sie soll ihm zeigen, daß auch in unserm Vaterlande der Jude zu Staatsämtern gelangen kann und in seiner öffentlichen Laufbahn nicht aufgehalten wird [...]. Es wird zwar für jetzt noch ausnahmsweise geschehen, allein es geschieht!“ (Burg 1998, 2-3).

Bei allem liberalen Fortschrittsoptimismus und Glauben an die Emanzipation der Juden im preußischen Staat spricht aus diesen Worten auch die Erkenntnis, als Jude mit Vorurteilen und Hindernissen kämpfen zu müssen, die von den Angehörigen der christlichen Mehrheit im Staat aufgerichtet worden waren und mit dem „Edikt betreffend die bürgerlichen Verhältnisse der Juden in dem Preußischen Staate“ vom 11. März 1812 noch lange nicht beseitigt waren. Das Edikt gewährte zwar die preußische Staatsbürgerschaft für die Juden, aber noch lange nicht ihre völlige Gleichberechtigung. Die Überwindung dieser Hindernisse gelang Burg selbst nur durch die Protektion des Prinzen August, der an den zentralen Wendepunkten von Burgs Lebens seinen Einfluss geltend machte, um ihm die nächste Beförderung zu erleichtern oder die nächste militärische Auszeichnung zu verleihen (vgl. hierzu Fischer 1968, 127-130). Meno Burg, trotz allem berechtigten Stolz auf die eigene Lebensleistung, war sich dessen durchaus bewusst, und er berichtet freimütig von den antijüdischen Erfahrungen, denen er im dienstlichen wie gesellschaftlichen Leben, in seinem Ringen um Anerkennung häufig ausgesetzt war. Dementsprechend sind seine Erwartungen in das preußische „Gesetz über die Verhältnisse der Juden“ vom 23. Juli 1847 auch ambivalent, und er weiß, dass die Angehörigen seiner ,Konfession‘ auch künftig mehr leisten müssen als die Angehörigen der christlichen Konfessionen, um dieselbe berufliche und gesellschaftliche Stellung zu erlangen: „Denn ungeachtet der jetzigen Gleichstellung wird der sich dem Staatsdienst widmende jüdische Jüngling noch manchen Kampf zu bestehen haben, manche Hin- 
dernisse mit Klugheit, Besonnenheit und möglichst kaltem Blut überwinden müssen, indem sich das leider noch hin und wieder zeigende Vorurteil doch nicht mit einem Male und urplötzlich beseitigen lässt“ (Burg 1998, 167-168).

Burgs Optimismus sollte sich jedoch schon bald als Trugschluss erweisen. Mit der Ablösung des bislang vorherrschenden, religiös motivierten Antijudaismus durch den ,wissenschaftlich' begründeten, rassistisch motivierten Antisemitismus seit den 1880er Jahren war es den deutschen Juden nicht einmal mehr theoretisch möglich, gleichberechtigte Aufnahme in die christliche Mehrheitsgesellschaft zu finden, obwohl sie de jure seit der Reichsgründung überall gleichgestellt waren.

Am deutlichsten wird dies in der Autobiographie des in Fürth 1873 geborenen Jakob Wassermann, der keine „eigentliche Lebensbeschreibung [...], sondern nur Darstellung eines schicksalhaften Konflikts“ (Wassermann 1994, 38) beabsichtigt. Er schrieb seine „Rechenschaft [...] über den problematischsten Teil meines Lebens, den [...] als deutscher Jude“ - „gedrängt von innerer Not der Zeit“ (Wassermann 1994, 7) - nach dem Ende des Krieges und veröffentlichte das Werk 1921. Seine Schreiberfahrung ist geprägt von einer tiefen Desillusionierung über die Möglichkeit des Gelingens der jüdischen Emanzipation durch Assimilation in Deutschland. Anlass dazu waren die zunehmenden antisemitischen Strömungen nach dem verlorenen Krieg, die eine Ursache sicherlich in der ,Judenzählung' im Krieg und der verfälschenden Berichterstattung über deren Ergebnisse in der jungen Weimarer Republik hatten. Die ,Judenzählung ' hatte ergeben, dass deutsche Juden prozentual ebenbürtig Kriegsdienst geleistet hatten, was in zahlreichen Publikationen geleugnet wurde und insofern einer antisemitischen Dolchstoßlegende Vorschub leistete. Folgerichtig schließt Wassermann sein bitteres Resümee über die Nutzlosigkeit aller und insbesondere seiner eigenen Emanzipations- und Assimilationsbestrebungen mit einem zehnfachen „Es ist vergeblich“, von dem hier das bittere Ende zitiert sei: „Es ist vergeblich, für sie zu leben und für sie zu sterben. Sie sagen: er ist ein Jude“ (Wassermann 1994, 122-123).

Wassermann argumentiert in seiner Autobiographie noch genau wie Meno Burg zwei Generationen zuvor ganz aus dem Geiste des Liberalismus, indem er versucht, seine individuellen Leistungen gegen die stereotypen Vorurteile der Mehrheitsgesellschaft in Stellung zu bringen und diese Vorurteile für seine Person als unbegründet zu entlarven. Damit legitimiert er den herrschenden Antisemitismus, ja er geht sogar so weit, eine „Blut- und Rasseverwandtschaft“ (Wassermann 1994, 103) aller Juden zu behaupten und sich, vor allem während seiner Zeit in Wien, für die Juden zu schämen: „Diese Scham steigerte sich manchmal bis zur Verzweiflung und bis zum Ekel.“ (Wassermann 1994, 103) Deshalb fühlt er sich als Kind und Jugendlicher vom Antisemitismus seiner Umwelt kaum getroffen, weil dieser „weniger die Person als die Gemeinschaft“ (Wassermann 1994, 12) angriff, der er selbst sich nicht zugehörig fühlt.

Die Erkenntnis der Unausweichlichkeit des Antisemitismus ist für Wassermann ein langwieriger Prozess, den Mein Weg als Deutscher und Jude ausführlich schil- 
dert, eine Erkenntnis, die erst bei dem erwachsenen, sich etablierenden Erfolgsschriftsteller reift. Vor allem die zahlreichen eingestreuten ,Gespräche“ mit anonym bleibenden ,Freunden“ dienen in essayartiger Form der Entfaltung seiner liberalistischen Argumentationskette gegen die antisemitischen Stereotype und enden für Wassermann allesamt unbefriedigend, weil er die Vorurteile seiner Gesprächspartner nicht abbauen kann. Mit seinen Argumenten bleibt Wassermann dabei aber weit hinter dem bereits im Berliner Antisemitismusstreit der 1880er Jahre erreichten Argumentationsstand zurück.

\section{Behauptete Subjektivität: Antisemitismus und Holocaust}

Wassermanns ,schicksalhafter Konflikt“ zwischen aufgezwungenem Judentum und frei gewählter gesellschaftlicher Zugehörigkeit bestimmt auch die Autobiographien der folgenden Generation, die sich nicht mehr nur in der liberalen Aufstiegsideologie einen Ausweg aus ihrem Judentum erhoffen, sondern in den verschiedenen Ideologien, die sich seit dem Ende des 19. Jahrhunderts über Europa verbreiteten, eine geistige ,Zugehörigkeit‘ (Greve 1994) suchen (vgl. Carmely 1981). Überschattet sind diese Texte von der Zäsur der nationalsozialistischen Vernichtungspolitik, die den Sozialisten und Zionisten als Bestätigung ihres Weges dient, den assimilierten Juden wie etwa Werner Kraft aber die Ausweglosigkeit ihrer Bestrebungen zeigt: „Schon im April 1933 verließ ich Deutschland. Seit 1934 lebe ich in Jerusalem. Es heißt, die Luft dieses Landes macht weise; das Exil wurde zur Heimat. Aber die Mühe des Anfangs war groß. Nicht nur die materiellen, auch die geistigen Lebensadern waren mir durchgeschnitten“ (Kraft 1973, 152). Wie beispielsweise in der Familie Gershom Scholems finden sich diese drei Möglichkeiten jüdischen Lebens in Deutschland oft in einer einzigen Familie vereint und wirken desintegrierend auf den Familienverband.

Die meisten deutschen Juden gingen den Weg der Assimilation weiter. Im Zentrum ihrer autobiographischen Texte stehen die frühe Hinwendung zur deutschen Kultur und Literatur sowie der meist erfolglose Versuch, sich in die christliche Mehrheitsgesellschaft nahtlos zu integrieren. Unter den Bedingungen von Antijudaismus und Antisemitismus konnte die angestrebte Integration durch Assimilation für den jüdischen Bevölkerungsanteil aber immer nur eine ,negative“ sein, d.h. eine Integration, die zwar eine Mimesis der Mehrheitsgesellschaft erlaubte, letztlich aber nur in einer Parallelwelt verwirklichbar war, die die Strukturen der christlichen bürgerlichen Gesellschaft nachahmte, von dieser aber durch unsichtbare Barrieren getrennt war. Dies erschwerte vor allem bei der Generation der nach dem Ersten Weltkrieg Geborenen die Identitätsfindung nachhaltig, weil wesentliche Angebote zur Identitätsbildung außerhalb des Judentums nicht zur Verfügung standen: „Trotz meiner Bemühungen, mich dem allgemeinen Verkehrston anzupassen - ich gehöre 
nicht dazu. Der Instinkt meiner Mitschüler verrät ihnen, daß ich mich bloß in Mimikry übe“ (Kunert 1997, 20).

Eine Gegenbewegung stellte der Zionismus dar, dessen Vertreter sich - gegen das Selbstverständnis noch der Elterngeneration - dem Judentum, seiner Geschichte, Kultur und Religion, bewusst zuwandten und perspektivisch die Auswanderung nach Palästina, damals noch britisches Mandatsgebiet, erstrebten. Von Berlin nach Jerusalem hat Gershom Scholem deshalb seine Autobiographie betitelt. Hier klingt die ,Lebensweg'-Metapher bereits im Titel an, die - trotz aller Widrigkeiten im antisemitischen Berlin seiner Jugend - eine stringente Persönlichkeitsentwicklung nahe legt und insofern einer gelungenen Gelehrtenautobiographie zu Recht voran steht.

Bemerkenswerterweise spielt der Sozialismus als Lebensform in den deutschjüdischen Autobiographien nur eine untergeordnete Rolle, obwohl zahlreiche jüdische Sozialisten autobiographische und Memoirenliteratur verfasst haben. Der Hauptgrund hierfür liegt sicherlich in der sozialistischen Gesellschaftsidee, die Nationen, Religionen und Klassen zu überwinden trachtet und deshalb einem wie auch immer gedeuteten Judentum keine lebensbestimmende Rolle, höchstens eine zu überwindende Zwischenstufe der Identitätsfindung zumessen kann.

Eine spezifische, wenn auch in ihrer Definition umstrittene Gattung des Schreibens über das eigene Leben stellt die autobiographisch motivierte ,Holocaustliteratur` dar, die das Überleben der nationalsozialistischen Vernichtungspolitik dokumentiert. In diesen Texten steht zumeist nicht ein autobiographisches Individuum und dessen Entwicklung im Zentrum, sondern die Zeugenschaft der Zustände im Lager, der Flucht oder des Überlebens in Verstecken (vgl. Taterka 1999). Sie soll deshalb an dieser Stelle nicht näher besprochen werden.

Natürlich stellt das Überleben des Holocaust auch in der jüdischen Autobiographie des 20. Jahrhunderts eine zentrales Thema dar (vgl. Lezzi 2001), das gegen alle inneren und äußeren Widerstände in das „weiter leben“ (Ruth Klüger) integriert werden muss, um nicht in den Status eines passiven Opfers oder in die Rolle eines bloßen Repräsentanten oder Zeugen eines kollektiven Schicksals gedrängt zu werden: „[I]ch komm nicht von Auschwitz her, ich stamm aus Wien. Wien lässt sich nicht abstreifen, man hört es an der Sprache, doch Auschwitz war mir so wesensfremd wie der Mond“ (Klüger 1992, 138).

\section{Die Überlebenden und die Nachgeborenen}

Die Verteidigung und Behauptung der eigenen Subjektivität sowie das Beharren auf der Einzigartigkeit stehen daher im Zentrum dieser autobiographischen Texte. Paradoxerweise hat gerade der Versuch der Auslöschung der Individualität der zur Vernichtung in den Lagern bestimmten Juden das Beharren der Autobiographen darauf gefördert (vgl. Malo 2009) und führt die jüdische Autobiographie aus der von 
Miething behaupteten spezifischen Differenz zur christlichen Autobiographik heraus. Dies zeigt sich etwa bei Ruth Klüger in einer starken Idiosynkrasie gegenüber Vereinnahmungstendenzen von deutscher Seite und gegen das "Shoah-Business“ der Gedenkstättenkultur, dem sie keinerlei Erkenntnis- oder moralischen Wert zubilligt. Sie sieht darin eine Nivellierung der Individualität der Opfer, die Gleichgültigkeit statt Empathie hervorruft und so letztlich wiederum zu einer Fremdbestimmung von Identität führt, die der Vielschichtigkeit der Identität der Autobiographen nicht gerecht wird, weil der Einzelne auf seinen kollektiven Holocaust-Überlebenden-Status reduziert wird, dem alle weitere Lebenserfahrung untergeordnet wird. Dagegen betont Klüger vor allem im zweiten Teil ihrer Autobiographie die Bedeutung einer weiteren, ihren späteren Lebens- und Bildungsgang genauso stark bestimmenden Diskriminierung, der sie als bildungs- und karriereorientierte Frau in einer männlich dominierten Gesellschaft ausgesetzt ist (Klüger 2008).

Die Fremdzuschreibung von Identität als wesentliches Problem der deutschsprachigen jüdischen Autobiographen bleibt ein Kontinuum der Gattung auch nach dem Holocaust. Allerdings steht jetzt nicht mehr die Abwehr antijüdischer oder antisemitischer Stereotype, die einen Zusammenschluss unter einer jüdischen Kollektividentität befördert haben, im Vordergrund des Rechtfertigungsdiskurses, sondern die Abwehr einer philosemitischen Umarmungsstrategie, die die Verbrechen der Vergangenheit an den Lebenden sühnen will und sich davon Absolution erhofft. Maxim Biller hat sein „Selbstporträt“ Der gebrauchte Jude betitelt und versucht, die daran von deutscher Seite geknüpften Erwartungen zu unterlaufen und seine publikumswirksame Wut zu legitimieren: „Alle Deutschen sind Idioten. Sie wollen etwas von mir, das ich ihnen nicht geben kann, und weil ich es ihnen nicht geben kann, sind sie sauer auf mich. Darum, dachte ich, werde ich ab jetzt auch auf sie sauer sein“ (Biller 2009, 31). Dagegen setzt er seine vielschichtige und vielfältig gebrochene Identität, die sich nicht auf das Judentum reduzieren lässt, dieses vielmehr nur als einen Ausdruck seiner Persönlichkeit betrachtet: „Ich will nicht Jude sein, weil man mich als Juden sieht. Ich will Jude, Mann, Schriftsteller sein, weil ich es bin“ (Biller 2009, 164). Derselbe Gedanke findet sich bei Ruth Klüger 1992, die in dem bei Simone Weil entlehnten Motto ihrer Autobiographie das subjektive Erleben über die vermeintlich objektiven Realitäten stellt: „Das Mißverhältnis zwischen der Einbildung und dem Sachverhalt ertragen. ,Ich leide.' Das ist besser als: ,Diese Landschaft ist häßlich“."

,Normalität‘ im deutsch-jüdischen, aber auch im jüdisch-deutschen Verhältnis herzustellen, wird wohl erst gelingen, wenn dieser Wunsch Billers und Klügers erfüllt ist und wenn auch deutsch-jüdische Autobiographen ,Lebensweg'-Diskurse (vgl. Vollers-Sauer 1993) führen können, die frei sind von einem wie auch immer gearteten deutsch-jüdischen (Sub-)Diskurs, der die eigene Identität vorwiegend durch die Abwehr von Fremdzuschreibungen herzustellen gezwungen ist. 


\section{Literaturverzeichnis}

\section{Autobiographien}

Biller, Maxim: Der gebrauchte Jude. Selbstporträt. Köln: Kiepenheuer \& Witsch, 2009.

Burg, Meno: Geschichte meines Dienstlebens. Erinnerungen eines jüdischen Majors der preußischen Armee. Mit einem Geleitwort von Ludwig Geiger. Vorwort von Hermann Simon. Teetz: Hentrich \& Hentrich, 1998.

[Glikl]: Die Memoiren der Glückel von Hameln. Autorisierte Übertragung nach der Ausgabe des Prof. Dr. David Kaufmann von Bertha Pappenheim. Wien: Meyer und Pappenheim, 1910.

Goethe, Johann Wolfgang: Dichtung und Wahrheit. Hrsg. von Peter Sprengel. München: Hanser, 1985.

Greve, Ludwig: Wo gehörte ich hin? Geschichte einer Jugend. Hrsg. und mit einem Nachbericht vers. von Reinhard Tgahrt. Frankfurt a.M.: Fischer, 1994.

Greve, Ludwig: Ein Besuch in der Villa Sardi. Porträts, Gedenkblätter, Reden. Hrsg. von Reinhard Tgahrt. Warmbronn: Keicher, 2001.

Klüger, Ruth: weiter leben. Eine Jugend. Göttingen: Wallstein, 1992.

Klüger, Ruth: Unterwegs verloren. Erinnerungen. Wien: Zsolnay, 2008.

Kraft, Werner: Spiegelung der Jugend. Mit einem Nachwort von Jörg Drews. Frankfurt a.M.: Suhrkamp, 1973.

Kunert, Günter: Erwachsenenspiele. Erinnerungen. München, Wien: Hanser, 1997.

Maimon, Salomon: Lebensgeschichte. Von ihm selbst geschrieben und hrsg. von Karl Philipp Moritz. Neu hrsg. von Zwi Batscha. Frankfurt a.M.: Jüdischer Verlag, 1995.

Richarz, Monika (Hrsg.): Jüdisches Leben in Deutschland. 3 Bde. Stuttgart: Deutsche VerlagsAnstalt, 1976-1982.

Scholem, Gershom: Von Berlin nach Jerusalem. Jugenderinnerungen. Aus dem Hebräischen von Michael Brocke und Andrea Schatz. Frankfurt a.M.: Suhrkamp, 1997.

Sperber, Manès: All das Vergangene ... Die Wasserträger Gottes. Die vergebliche Warnung. Wien u.a.: Europaverlag, 1983.

Wassermann, Jakob: Mein Weg als Deutscher und Jude. München. Deutscher Taschenbuch-Verlag, 1994.

\section{Forschungsliteratur}

Carmely, Klara Pomeranz: Das Identitätsproblem jüdischer Autoren im deutschen Sprachraum. Von der Jahrhundertwende bis zu Hitler. Königstein i.Ts.: Scriptor, 1981.

Fischer, Horst: Judentum, Staat, Heer in Preußen im frühen 19. Jahrhundert. Zur Geschichte der staatlichen Judenpolitik. Tübingen: Mohr (Siebeck), 1968.

Gasser, Peter: „Autobiographie und Autofiktion. Einige begriffskritische Bemerkungen“. Pellin, Elio; Weber, Ulrich (Hrsg.): „....all diese fingierten, notierten, in meinem Kopf ungefähr wieder zusammengesetzten Ichs“. Autobiographie und Autofiktion. Göttingen: Wallstein, 2012, 13-27.

Glagau, Hans: Die moderne Selbstbiographie als historische Quelle. Eine Untersuchung. Marburg: Elwert, 1903. Auszugsweise wiederabgedruckt in: Niggl 1998, 55-71.

Glasenapp, Gabriele von: „,Wir haben das nicht unversehrt überstanden'. Jüdische Kindheit und Jugend während des Dritten Reiches im Spiegel autobiographischer Texte“. Glasenapp, Gabriele von; Ewers, Hans-Heino (Hrsg.): Kriegs- und Nachkriegskindheiten. Studien zur literarischen Erinnerungskultur für junge Leser. Frankfurt a.M. u.a.: Peter Lang, 2008, 127-149. 
Günter, Manuela: Anatomie des Anti-Subjekts. Zur Subversion autobiographischen Schreibens bei Siegfried Kracauer, Walter Benjamin und Carl Einstein. Würzburg: Königshausen \& Neumann, 1996.

Holdenried, Michaela: Im Spiegel ein anderer. Erfahrungskrise und Subjektdiskurs im modernen autobiographischen Roman. Heidelberg: Winter, 1991.

Holdenried, Michaela: Autobiographie. Stuttgart: Reclam, 2000.

Kilcher, Andreas: „Was ist ,deutsch-jüdische Literatur'? Eine historische Diskursanalyse“. Weimarer Beiträge 45 (1999), 485-517.

Lehmann, Jürgen: Bekennen, erzählen, berichten. Studien zu Theorie und Geschichte der Autobiographie. Tübingen: Niemeyer, 1988.

Lejeune, Philipe: „Der autobiographische Pakt“. Wieder abgedruckt in: Niggl 1998, 215-257.

Lezzi, Eva: Zerstörte Kindheit. Literarische Autobiographien zur Shoah. Köln, Weimar, Wien: Böhlau, 2001.

Malo, Markus: Behauptete Subjektivität. Eine Skizze zur deutschsprachigen jüdischen Autobiographie im 20. Jahrhundert. Tübingen: Niemeyer, 2009.

de Man, Paul: „Autobiographie als Maskenspiel“. Ders.: Die Ideologie des Ästhetischen. Hrsg. von Christoph Menke. Frankfurt a.M.: Suhrkamp, 1993, 131-146.

Miething, Christoph: „Gibt es jüdische Autobiographien?“ Miething, Christoph (Hrsg.): Zeitgenössische jüdische Autobiographie. Tübingen: Niemeyer, 2003, 43-73.

Montrose, Louis A.: „Die Renaissance behaupten. Poetik und Politik der Kultur“. Baßler, Moritz (Hrsg.): New Historicism. Literaturgeschichte als Poetik der Kultur. Frankfurt a.M.: Fischer, 1995, 60-93.

Müller, Klaus-Detlef: Autobiographie und Roman. Studien zur literarischen Autobiographie der Goethezeit. Tübingen: Niemeyer, 1976.

Neumann, Bernd: Identität und Rollenzwang. Zur Theorie der Autobiographie. Frankfurt a.M.: Athenäum, 1970.

Niggl, Günter: Geschichte der deutschen Autobiographie im 18. Jahrhundert. Theoretische Grundlegung und literarische Entfaltung. Stuttgart: Metzler, 1977.

Niggl, Günther (Hrsg.): Die Autobiographie. Zu Form und Geschichte einer literarischen Gattung. Studienausgabe, 2., um ein Nachwort zur Neuausgabe und einen bibliograph. Nachtrag erg. Aufl. Darmstadt: Wissenschaftliche Buchgesellschaft, 1998.

Sandberg, Beatrice: „Erinnerte und erfundene Erfahrung. Autobiographisches Schreiben als subjektive Geschichtsschreibung“. Platen, Edgar (Hrsg.): Erinnerte und erfundene Erfahrung. Zur Darstellung von Zeitgeschichte in deutschsprachiger Gegenwartsliteratur. München: ludicium, 2000, 146-161.

Sill, Oliver: Zerbrochene Spiegel. Studien zur Theorie und Praxis modernen autobiographischen Erzählens. Berlin: De Gruyter, 1991.

Taterka, Thomas: Dante Deutsch. Studien zur Lagerliteratur. Berlin u.a.: Erich Schmidt, 1999.

Vollers-Sauer, Elisabeth: Prosa des Lebensweges. Literarische Konfigurationen selbstbiographischen Erzählens am Ende des 18. und 19. Jahrhunderts. Stuttgart: M \& P, 1993.

Wagner-Egelhaaf, Martina: Autobiographie. Stuttgart, Weimar: Metzler, 2000.

White, Hayden: Auch Klio dichtet oder Die Fiktion des Faktischen. Studien zur Tropologie des historischen Diskurses. Einf. von Reinhart Koselleck. Stuttgart: Klett-Cotta, 1986.

Yerushalmi, Yosef Hayim: Zachor, erinnere Dich! Jüdische Geschichte und jüdisches Gedächtnis. Berlin: Wagenbach, 1996. 